\title{
Excited State Effective Masses
}

\section{George T. Fleming*}

Yale University

E-mail: George.Fleming@Yale.edu

\section{Saul D. Cohen}

Thomas Jefferson National Accelerator Facility

E-mail: sdcohen@jlab.org

\section{Huey-Wen Lin}

Thomas Jefferson National Accelerator Facility

E-mail: hwlinejlab.org

\section{Victor Pereyra}

Weidlinger Associates Inc., Mountain View, CA

E-mail: victor@wai.com

The effective mass description of Euclidean time hadron correlation functions is extended to incorporate multiple excited states and multiple correlation functions. In general, the effective masses will be determined by finding the roots of some polynomial.

The XXV International Symposium on Lattice Field Theory

July 30-4 August 2007

Regensburg, Germany

\footnotetext{
*Speaker.
} 
In Lattice QCD with infinite temporal extent, typical hadronic Euclidean time correlation functions can be described by the model function

$$
\begin{gathered}
C\left(\vec{p}, t_{n}\right)=\sum_{m=1}^{M} A_{m}(\vec{p}) \exp \left[-\left(t_{0}+n a\right) E_{m}(\vec{p})\right] \\
n \geq 0, \quad A_{m}, E_{m} \in \mathbb{R}, \quad 0 \leq E_{1} \leq E_{2} \leq \cdots \leq E_{M} .
\end{gathered}
$$

In general, the correlation function computed on $N=2 M$ time-slices $t_{n}$ will admit a solution of $M$ energies $E_{m}$ and amplitudes $A_{m}$. The problem to solve is the nonlinear system of equations $\mathbf{y}=\mathbf{V}(x) \mathbf{a}$ :

$$
\left[\begin{array}{c}
y_{1} \\
y_{2} \\
y_{3} \\
\vdots \\
y_{2 M}
\end{array}\right]=\left[\begin{array}{cccc}
1 & 1 & \cdots & 1 \\
x_{1} & x_{2} & \cdots & x_{M} \\
x_{1}^{2} & x_{2}^{2} & \cdots & x_{M}^{2} \\
\vdots & \vdots & \ddots & \vdots \\
x_{1}^{2 M-1} & x_{2}^{2 M-1} & \cdots & x_{M}^{2 M-1}
\end{array}\right]\left[\begin{array}{c}
a_{1} \\
\vdots \\
a_{M}
\end{array}\right]
$$

for $x_{m}=\exp \left[-a E_{m}(\vec{p})\right]$ and $a_{m}=A_{m}(\vec{p}) \exp \left[-t_{0} E_{m}(\vec{p})\right]$ where $y_{n}=C\left(\vec{p}, t_{n}\right) . \mathbf{V}(x)$ is known as $2 M \times M$ rectangular Vandermonde matrix.

By inspection, it appears the problem is of polynomial degree $2 M$ and thus should not admit a general closed form solution in terms of radicals by the Abel-Ruffini theorem [1, 2] for $M>2$. The $M=1$ solution is simple to compute and is widely known in the Lattice QCD literature as the effective mass solution. Note already that the simple effective mass problem is linear and has only one solution, suggesting that the polynomial degree is actually of order $M$.

The $M=2$ solution was explicitly constructed in the Lattice QCD context by one of the authors [3] and was independently constructed some time later by others [4]. In the absence of noise in the $y_{n}$, the general solution was known as early as 1795 to Prony [5] as shown below. It was noted [3] that the problem, when reduced, required only the solution of a quadratic equation and so it was conjectured that the general problem of size $M$ could be reduced to a polynomial equation in one variable of degree $M$.

An efficient algorithm has been available for some time for solving square Vandermonde systems [6] by making them upper triangular. This approach works equally well for rectangular Vandermonde systems as in Eq. (2). Furthermore, this approach reveals why the solution for the energies $E_{m}$ can be found without solving for the amplitudes $A_{m}$ and why the problem is of polynomial degree $M$.

As a first step we transform the system so that $\mathbf{V}(x)$ is in upper triangular form [6] by premultiplying by the lower $2 M \times 2 M$ bi-diagonal matrices:

$$
L_{m}(x)=\left[\begin{array}{ccccccc}
1 & & & & & & \\
0 & \ddots & & & & & \\
& \ddots & 1 & & & & \\
& & 0 & 1 & & & \\
& & x_{m} & -1 & & \\
& & & & \ddots & \ddots & \\
& & & & & x_{m}-1
\end{array}\right],
$$


where the first -1 on the diagonal appears in the $m+1$ row and column.

To reduce the $M=2$ problem, we pre-multiply by two factors of the bi-diagonal matrices of Eq. (3) to find the reduced equation $\mathbf{L}_{\mathbf{2}} \mathbf{L}_{\mathbf{1}} \mathbf{y}=\mathbf{L}_{\mathbf{2}} \mathbf{L}_{\mathbf{1}}$ Va. By introducing the auxiliary quantities:

$$
\begin{aligned}
& \alpha_{i}=x_{1} y_{i-1}-y_{i} \quad(2 \leq i \leq 2 M) \\
& \beta_{j}=x_{2} \alpha_{j-1}-\alpha_{j} \quad(3 \leq j \leq 2 M),
\end{aligned}
$$

the reduced system becomes:

$$
\begin{aligned}
& y_{1}=a_{1}+a_{2} \\
& \alpha_{2}=a_{2}\left(x_{1}-x_{2}\right) \\
& \beta_{3}=0 \\
& \beta_{4}=0 .
\end{aligned}
$$

The first half of the equations, Eqs. (6) and (7), involve both the amplitudes $A_{1}, A_{2}$ and the energies $E_{1}, E_{2}$ but the second half involve only the energies. It will be true for any $M$, in general, that the last $M$ equations can be solved first to find all the energies. Once all the energies are known, the first $M$ equations form a square upper triangular system that can be solved efficiently by backward substitution to find the amplitudes.

To see that Eqs. (6)-(9) yield the known solution [3], first substitute Eq. (5) and eliminate $x_{2}$ from Eqs. (8)-(9) to find

$$
\alpha_{2} \alpha_{4}-\alpha_{3}^{2}=0, \quad \text { or } \quad\left|\begin{array}{ll}
\alpha_{2} & \alpha_{3} \\
\alpha_{3} & \alpha_{4}
\end{array}\right|=0,
$$

where we note that the 1.h.s. is the determinant of a $2 \times 2$ Hankel matrix or perhaps the minor of a larger Hankel matrix. After substituting Eq. (4), this gives the known quadratic equation

$$
\left(y_{2}^{2}-y_{1} y_{3}\right) x_{1}^{2}+\left(y_{1} y_{4}-y_{2} y_{3}\right) x_{1}+\left(y_{3}^{2}-y_{2} y_{4}\right)=0 .
$$

Note that this can also be written

$$
\left|\begin{array}{ll}
y_{1} & y_{2} \\
y_{2} & y_{3}
\end{array}\right| x_{1}^{2}-\left|\begin{array}{ll}
y_{1} & y_{2} \\
y_{3} & y_{4}
\end{array}\right| x_{1}+\left|\begin{array}{ll}
y_{2} & y_{3} \\
y_{3} & y_{4}
\end{array}\right|=0,
$$

where the coefficients are not determinants of Hankel matrices but minors of a single Hankel matrix. So, it can be written even more compactly as

$$
\left|\begin{array}{ll:l}
y_{1} & y_{2} & 1 \\
y_{2} & y_{3} & x_{1} \\
y_{3} & y_{4} & x_{1}^{2}
\end{array}\right|=0
$$

where the left block is a Hankel matrix and the right block is a Vandermonde matrix.

Following the same procedure, the $M=3$ polynomial can be written

$$
\left|\begin{array}{lll:l}
y_{1} & y_{2} & y_{3} & 1 \\
y_{2} & y_{3} & y_{4} & x_{1} \\
y_{3} & y_{4} & y_{5} & x_{1}^{2} \\
y_{4} & y_{5} & y_{6} & x_{1}^{3}
\end{array}\right|=0
$$


and solved using the method of Scipione del Ferro and Tartaglia [7], and the $M=4$ polynomial can be written

$$
\left|\begin{array}{llll:c}
y_{1} & y_{2} & y_{3} & y_{4} & 1 \\
y_{2} & y_{3} & y_{4} & y_{5} & x_{1} \\
y_{3} & y_{4} & y_{5} & y_{6} & x_{1}^{2} \\
y_{4} & y_{5} & y_{6} & y_{7} & x_{1}^{3} \\
y_{5} & y_{6} & y_{7} & y_{8} & x_{1}^{4}
\end{array}\right|=0
$$

and solved using the method of Ferrari [7].

Although there are no general solutions in radicals for polynomials higher than quartic order, there are numerical methods for finding the roots of polynomials of any order. The general form for the polynomial follows from Eqs. (13), (14) and (15):

$$
|\mathscr{H}|=\left|\begin{array}{cccc:c}
y_{1} & y_{2} & \cdots & y_{M} & 1 \\
y_{2} & y_{3} & \cdots & y_{M+1} & x_{1} \\
\vdots & \vdots & \ddots & \vdots & \vdots \\
y_{M+1} & y_{M+2} & \cdots & y_{2 M} & x_{1}^{M}
\end{array}\right|=0 .
$$

Prony [5] showed that problems in the form of Eq. (1) implied the following system of equations $\mathbf{y}=\mathbf{H}(y) \mathbf{p}$

$$
\left[\begin{array}{c}
y_{1} \\
y_{2} \\
\vdots \\
y_{M}
\end{array}\right]=-\left[\begin{array}{ccc}
y_{2} & \cdots & y_{M+1} \\
y_{3} & \cdots & y_{M+2} \\
\vdots & \ddots & \vdots \\
y_{M+1} & \cdots & y_{2 M}
\end{array}\right]\left[\begin{array}{c}
p_{1} \\
p_{2} \\
\vdots \\
p_{M}
\end{array}\right]
$$

where the $M \times M$ matrix $\mathbf{H}(y)$ has the special structure of a Hankel matrix and the components $p_{m}$ of $\mathbf{p}$ are the coefficients of a polynomial

$$
P(x)=\prod_{m=1}^{M}\left(x-x_{m}\right)=\sum_{m=0}^{M} p_{m} x^{m} \quad\left(p_{0}=1\right) .
$$

The Prony-Yule-Walker method (or just Prony's method, for short) [5, 8, 9] solves Eq. (17) to find the coefficients and then finds the $M$ roots of the polynomial in Eq. (18). The amplitudes are determined by substituting the roots into Eq. (2) and solving it. Note again that using $2 M$ timeslices of correlation function data to determine $M$ effective masses is a problem of polynomial order $M$. The general conditions under which the solutions of the Hankel and Vandermonde systems coincide is presented in [10]. A simple demonstration that both solutions are the same under some basic assumptions will be provided elsewhere.

When constructing correlation functions in Lattice QCD, care is taken to ensure that the correlation function transforms irreducibly under the symmetries of the lattice space group [11, 12]. For the model function, as in Eq. (1), this implies that the amplitudes depend on the details of the specific correlation function but that the energies depend only on the irreducible representation. Since it is common in Lattice QCD simulations to compute at least two distinct correlation functions for each irreducible representation, effective mass solutions which combine data from multiple correlators are also possible. 
Assume that there are $K$ correlation functions available as in Eq. (1) that differ only in their amplitudes:

$$
\begin{gathered}
C_{k}\left(\vec{p}, t_{n}\right)=\sum_{m=1}^{M} A_{k m}(\vec{p}) \exp \left[-\left(t_{0}+n a\right) E_{m}(\vec{p})\right] \\
n \geq 0, \quad A_{k m}, E_{m} \in \mathbb{R}, \quad 0 \leq E_{1} \leq E_{2} \leq \cdots \leq E_{M} .
\end{gathered}
$$

Data from the same $N$ time slices will be used in the following from each correlation function to construct $M$ effective masses. Under this assumption the condition that there will be equal number of data points as unknowns is $K N=(K+1) M$. Previously shown are the three solutions that satisfy the condition for $K=1$, up to quartic order. There are four more solutions for $K>1:(K, M, N)=$ $(2,2,3),(2,4,6),(3,3,4)$ and $(4,4,5)$. Relaxing the assumption that the same number of time slices are used from each correlation function will allow for more possibilities up to quartic order. It is straightforward to generalize to these cases if desired.

The nonlinear equations to solve have a block structure:

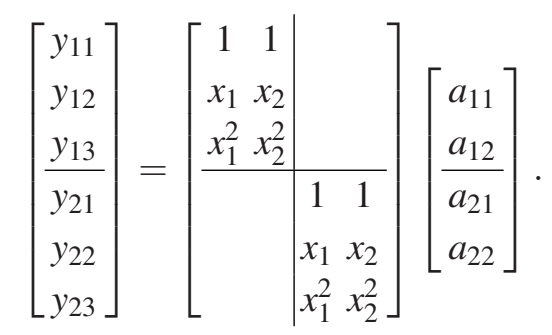

Here, the indices for $y_{k n}, x_{m}$ and $a_{k m}$ are in the ranges $1 \leq k \leq K, 1 \leq m \leq M$ and $1 \leq n \leq N$. To reduce the system we extend Eq. (3) to block form with $K$ identical blocks $L_{m}(x)$ on the diagonal. The reduced equations are

$$
\begin{aligned}
y_{k 1} & =a_{k 1}+a_{k 2} \\
\alpha_{k 2} & =\left(x_{1}-x_{2}\right) a_{k 2} \\
\beta_{k 3} & =0
\end{aligned}
$$

$$
(1 \leq k \leq 2)
$$

where we have added an additional index $k$ to the auxiliary quantities defined in Eqs. (4) and (5). Substituting for $\beta_{k 3}$ in Eqs. (23) and eliminating $x_{2}$ gives the equation:

$$
\left|\begin{array}{ll}
\alpha_{12} & \alpha_{22} \\
\alpha_{13} & \alpha_{23}
\end{array}\right|=0,
$$

where we've written the equation as a minor of some matrix, following our previous experience, whose structure is not yet clear. Substituting for $\alpha_{k n}$ gives a quadratic equation in $x_{1}$ in determinant form:

$$
\left|\begin{array}{c:c:c}
y_{11} & y_{21} & 1 \\
y_{12} & y_{22} & x_{1} \\
y_{13} & y_{23} & x_{1}^{2}
\end{array}\right|=0 .
$$

Similar equations can be derived for $(K, M, N)=(2,4,6),(3,3,4)$ and $(4,4,5)$. 
The general form of the polynomial equation can be inferred by studying solved examples as in Eq. (25). Define $K$ Hankel matrices $H_{k}^{N \times M_{k}}$ for each of the correlation functions with the constraints $\sum_{k=1}^{K} M_{k}=M$ and $N=M+1$. Then the general form of the polynomial equation is

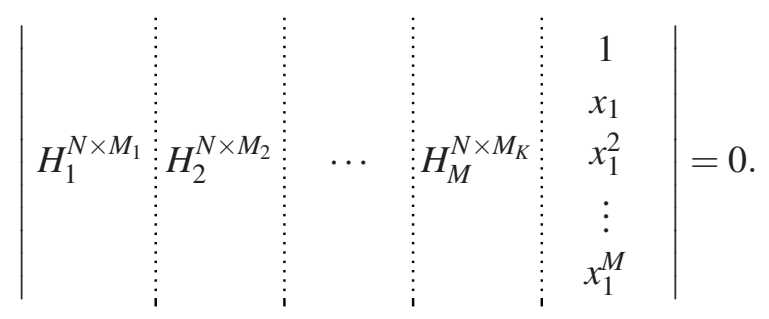

As previously discussed, each Hankel matrix is generally of full column rank, and, if the correlation functions are linearly independent, then the columns of different Hankel matrices are also linearly independent. So, Eq. (26) will only be satisfied for discrete values of $x_{1}$ corresponding to the roots of the polynomial.

In practical Lattice QCD calculations, the temporal extent is finite so the choice of temporal boundary conditions affects hadronic correlation functions near the boundary. For simplicity, starting from Eq. (1), set $t_{0}=0$ and identify the points $t_{0}=0$ and $t_{N}=N a$ which can be done using modular arithmetic, i.e. $t_{n}=(n \bmod N)$ a. For anti-periodic boundary conditions, the typical hadronic Euclidean time correlation function is described by the model function

$$
\begin{gathered}
C\left(\vec{p}, t_{n}\right)=\sum_{m=1}^{M}\left\{A_{m}(\vec{p}) \exp \left[-(n \bmod N) a E_{m}(\vec{p})\right]+(-1)^{B} A_{m}^{*}(\vec{p}) \exp \left[-(N-n \bmod N) a E_{m}^{*}(\vec{p})\right]\right\} \\
n \geq 0, \quad A_{m}, A_{m}^{*}, E_{m}, E_{m}^{*} \in \mathbb{R}, \quad 0 \leq E_{1} \leq E_{2} \leq \cdots \leq E_{M}, \quad 0 \leq E_{1}^{*} \leq E_{2}^{*} \leq \cdots \leq E_{M}^{*} .
\end{gathered}
$$

For periodic boundary conditions, set $(-1)^{B} \rightarrow 1$. For mesons, $B=0$ but more importantly $A_{m}=$ $A_{m}^{*}$ and $E_{m}=E_{m}^{*}$ which is not true for baryons $(B=1)$. So, baryon correlation functions represent $M$ states propatating to the left and $M$ different states propagating to the right for a total of $2 M$ states.

Meson correlation functions represent the same $M$ states propagating to the right and left but time-reversal symmetry relates $t_{n}$ and $t_{N-n}$ so that the half as many states are represented but by only half as many independent timeslices. As shown in [3], this can be made explicit by writing the meson correlation function as

$$
C\left(\tau_{n}\right)=\sum_{m=1}^{M} A_{m} \exp \left(-a N E_{m} / 2\right) \cosh \left(a n E_{m}\right), \quad \tau_{n}=(n-N / 2) a
$$

To write this result in the Vandermonde form of Eq. (2), define the variables

$$
a_{m}=A_{m} \exp \left(-a N E_{m} / 2\right), \quad x_{m}=\cosh \left(a E_{m}\right), \quad y_{n}=\frac{1}{2^{n-1}} \sum_{j=0}^{n-1}\left(\begin{array}{c}
n-1 \\
j
\end{array}\right) C\left(\tau_{n-2 j-1}\right) .
$$

For fermions lacking a positive single timeslice transfer matrix, e.g. domain wall fermions with $1<m_{0}<2$ [13], staggered fermions or even Wilson fermions with $0<r<1$ [14], oscillitory states will appear in the Euclidean time correlation functions

$$
C\left(\vec{p}, t_{n}\right)=\sum_{m=1}^{M}(-1)^{m n} A_{m}(\vec{p}) \exp \left[-\left(t_{0}+n a\right) E_{m}(\vec{p})\right]
$$



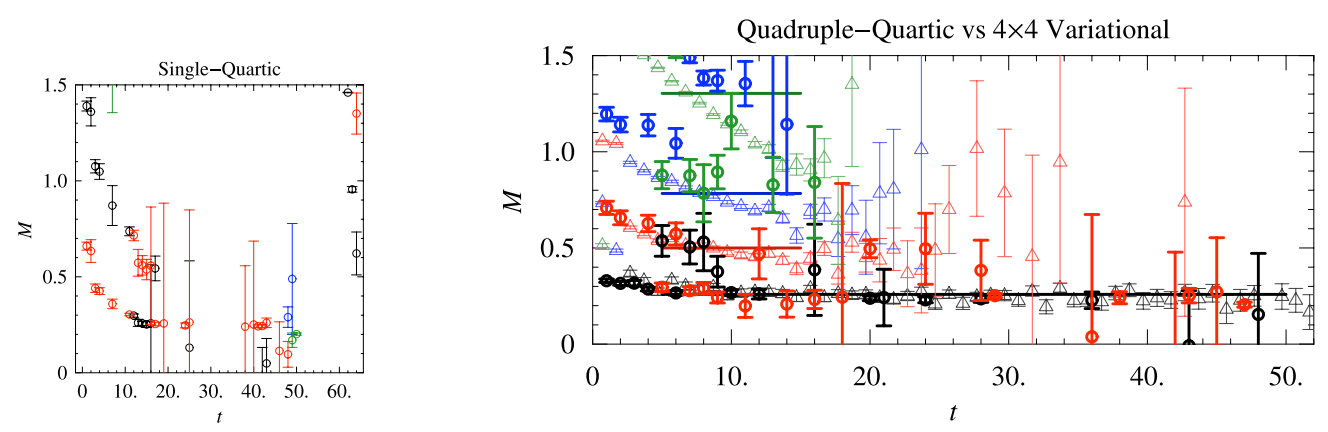

Figure 1: Left: Effective masses extracted from an ensemble of nucleon correlator using $(K, M, N)=$ $(4,1,8)$ solution. Colors indicate different roots of the polynomial in the order (lowest to highest) black, red, blue, green. Right: Effective masses on the same ensembles but using four correlators with different smearing radii on the source and sink. Roots are from the $(K, M, N)=(4,4,5)$ solution. Triangles indicate the results of a $4 \times 4$ variational analysis using sixteen correlators with all possible combinations of different smearings at the source and sink.

$$
n \geq 0, \quad A_{m}, E_{m} \in \mathbb{R}, \quad 0 \leq E_{1} \leq E_{3} \leq \cdots \leq E_{M-1}, \quad 0 \leq E_{2} \leq E_{4} \leq \cdots \leq E_{M} .
$$

Such states do appear as negative roots of the polynomial, Eq. (16) or (26), and should be considered non-spurious as dictated by the transfer matrix.

In summary, non-spurious polynomial roots may be negative depending on the transfer matrix and may have magnitude $|x|>1$ for backward propagating baryonic states. Folding mesonic correlation functions eliminates the backward propagating states, so $|x|<1$ for mesons.

\section{References}

[1] N. H. Abel, J. reine angew. Math. 1, 65 (1826).

[2] P. Ruffini, Teoria generale delle equazioni, in cui si dimostra impossibile la soluzione algebraica delle equazioni generali di grad superiore al quarto, di Paolo Ruffini (Nella stamperia di S. Tommaso d'Aquino, Bologna, 1799).

[3] G. T. Fleming, in $Q C D$ and Numerical Analysis III, edited by A. Boriçi, A. Frommer, B. Joó, A. Kennedy, and B. Pendleton (Springer-Verlag, 2005), no. 47 in Lecture Notes in Computational Science and Engineering, pp. 143-152, ISBN 3-540-21257-4, hep-lat/ 0403023.

[4] D. Guadagnoli, M. Papinutto, and S. Simula, Phys. Lett. B604, 74 (2004), hep-lat/ 0409011 .

[5] G. C. F. M. R. de Prony, J. Ecole Poly. 1, 24 (1795), partial translation available in [10].

[6] Å. Björck and V. Pereyra, Math. Comput. 24, 893 (1970).

[7] G. Cardano, Ars Magna (1545), http://en.wikipedia.org/wiki/Cubic_ equation. 
[8] G. Walker, Proc. Roy. Soc. Lond. A131, 518 (1931).

[9] G. U. Yule, Phil. Trans. Roy. Soc. Lond. A226, 267 (1927).

[10] D. Vandevoorde, Ph.D. thesis, Rensselaer Polytechnic Institute, Troy, New York, USA (1996), http://wwwlib.umi.com/dissertations/fullcit/9806112.

[11] S. Basak et al. (Lattice Hadron Physics (LHP)), Phys. Rev. D72, 094506 (2005), hep-lat / 0506029 .

[12] S. Basak et al. (Lattice Hadron Physics (LHP)), Phys. Rev. D72, 074501 (2005), hep-lat/ 0508018.

[13] S. Syritsyn and J. W. Negele, PoS LATTICE 2007, 78 (2007), arXiv:0710. 0425 [hep-lat].

[14] M. Creutz, Phys. Rev. D35, 1460 (1987). 